\title{
Coherent generation of EPR-entangled light pulses mediated by a single trapped atom
}

\author{
Giovanna Morigi \\ Grup d'Optica, Departament de Fisica, Universitat Autonoma de Barcelona, 08193 Bellaterra, Spain \\ Jürgen Eschner \\ ICFO, Institut de Ciències Fotóniques, 08860 Castelldefels, Barcelona, Spain \\ Stefano Mancini and David Vitali \\ CNISM and Dipartimento di Fisica, Università di Camerino, 62032 Camerino, Italy
}

(Received 23 December 2005; published 30 March 2006)

\begin{abstract}
We show that a single, trapped, laser-driven atom in a high-finesse optical cavity allows for the quantumcoherent generation of entangled light pulses on demand. We report the detailed description of schemes for generating simultaneous and temporally separated pulse pairs, presented in [G. Morigi et al., Phys. Rev. Lett., 96, 023601 (2006)]. The mechanical effect of the laser excitation on the quantum motion of the cold trapped atom mediates the entangling interaction between two cavity modes and between the two subsequent pulses, respectively. The entanglement is of EPR-type, and its degree can be controlled through external parameters. At the end of the generation process the atom is decorrelated from the light field. Possible experimental implementations of the proposals are discussed.
\end{abstract}

DOI: 10.1103/PhysRevA.73.033822

PACS number(s): 42.50.Dv, 32.80.Qk, 32.80.Lg

\section{INTRODUCTION}

One of the most intriguing features of quantum mechanics is the possibility of entangling physical systems, which has both fundamental and practical implications. In particular, entanglement has been recognized as a valuable resource for quantum information processing and for cryptography. In this context, two approaches have been developed, one based on discrete variables, the other using continuous variables. The main motivation to deal with continuous variables originates from practical considerations: efficient implementation of the essential steps of quantum information processing are achievable in quantum optics, utilizing the continuous quadrature variables of the quantized electromagnetic field [1]. In the continuous variable setting, Gaussian states play a prominent role, and when considering bipartite Gaussian systems, entangled states are synonymous to two-mode squeezed states [1], and their entanglement is equivalent to the position-momentum entanglement originally considered by Einstein, Podolsky, and Rosen (EPR) [2].

Conventionally, two-mode squeezed states emerge from the nonlinear optical interaction of a laser with a crystal, i.e., from parametric amplification or oscillation. As such, the phenomenon is the result of many-atom dynamics (often described by a simple nonlinear polarizability model). Then, an interesting question is whether analogous macroscopic nonlinear phenomena can emerge as well from the quantum dynamics of a single atom.

Recently, several experimental realizations have accessed novel regimes of engineering atom-photon interaction and opened promising perspectives for implementing controlled nonlinear dynamics with simple quantum optical systems. Examples are entangled light generation in atomic ensembles $[3,4]$, atomic memory for quantum states of light [5-7], entanglement between a single atom and its emitted photon $[8,9]$, entanglement of remote ensembles [10], one-atom la- ser $[11,12]$, mechanical forces of single photons on single atoms [13-15], controlled interaction of a trapped ion and a cavity $[16,17]$, controlled single-photon generation [18-20], as well as quantum state and entanglement engineering in the microwave regime [21].

In this work we investigate the realization of an optical parametric amplifier based on a single cold trapped atom inside a high-finesse optical cavity and driven by a short laser pulse, as sketched in Fig. 1. We show theoretically that this system allows for the controlled, quantum-coherent generation of entangled light pulses by exploiting the mechanical effects of atom-photon interaction. The pulses can contain many $(\gg 1)$ photons, and their entanglement is of continuous-variable (or EPR) type.

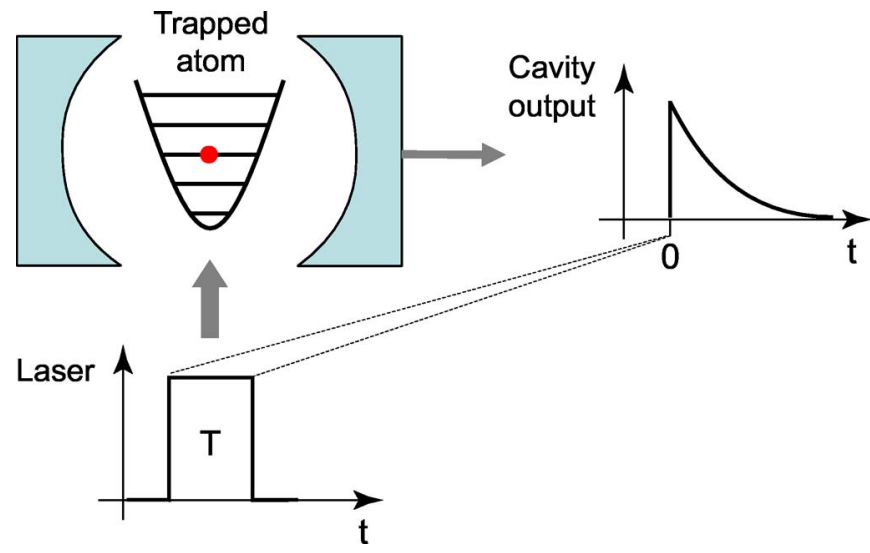

FIG. 1. (Color online) A trapped atom (ion) is confined inside a good resonator and is driven by a laser pulse of duration $T$ which propagates orthogonally to the cavity axis. By coupling the external atomic degrees of freedom to the cavity dynamics the pulse prepares the cavity field in a nonclassical state, which is transmitted to the output by cavity decay. A basic condition for the validity of these dynamics is $\kappa T \ll 1$, whereby $\kappa$ is the cavity decay rate. 
We present the detailed study of two schemes, which have been proposed in Ref. [22], and discuss in particular experimental conditions for their realization. The first scheme requires a bichromatic cavity interacting with the atomic dipole. Here, we show that-after short coherent excitation of the atom by an external laser beam-the cavity emits a bichromatic pulse of two-mode squeezed, i.e., entangled light. The second scheme relies on the interaction between the dipole and one cavity mode. Here, creation of two subsequent, entangled pulses at the cavity output is accomplished by using two temporally separated excitation pulses, with the quantum state of the atomic motion serving as intermediate memory which mediates the entanglement between the first and second pulse at the cavity output. In both cases entanglement is found on time scales of the order of the cavity decay time. In particular, noise reduction below $10 \%$ of the shot-noise level in the relative amplitude fluctuations of the two light fields is derived for an experimentally accessible set of parameters. Variation of the coupling parameters between atom and light allows for tuning the degree of entanglement between the cavity modes. Moreover, the emitted light pulses are decorrelated from the atom, i.e., at the end of the process the atom carries no memory of the interaction.

The scheme for generating a bichromatic pulse of twomode squeezed light applies concepts developed for macroscopic oscillators $[23,24]$ to a single quantum optical system, by exploiting the coupling between internal (electronic) and external (motional) degrees of freedom. Such coupling is negligible in macroscopic systems, but significant in atomic systems, thus rendering the dynamics far more accessible. The proposed scheme differs fundamentally from existing methods for generating pulsed squeezing [25] or intense pulses of polarization-entangled photons [26] which employ nonlinear crystals driven by a pulsed pump: in our case the microscopic nature of the medium allows for full coherent control of the light-matter quantum correlations and of the final quantum state of the generated light.

This study is connected to ideas of mapping quantum states of atoms onto light inside a cavity [27], to their implementation for establishing entanglement between distant atoms [28], and to recent experimental and theoretical studies on quantum correlations in the light scattered by atoms [3-5,7,29-31].

The paper is organized as follows. In Sec. II A we define the master equation governing the coherent and dissipative dynamics of the internal and motional degrees of freedom of the laser-driven atom and of the two relevant modes of the cavity coupling to the atom. In Sec. II B we derive the approximate Hamiltonian effecting the dynamics which lead to the generation of entanglement between the cavity modes, and in Sec. II C we discuss the dynamics it generates. In Sec. II D we introduce the field at the cavity output and in Sec. II E we investigate its quantum correlations. Section II F discusses the possibility of experimentally realizing the proposed scheme, i.e., the required experimental setup and parameters which would allow to observe the dynamics. Then, in Sec. III we discuss the (conceptually simpler) case of creating temporally separated, entangled pulses in one and the same cavity mode. The conclusions are drawn in Sec. IV, and in the appendix details of the calculations at the basis of the results in Sec. II E are provided.

\section{SIMULTANEOUS BICHROMATIC PULSES}

In this section we present a scheme for the simultaneous generation of bichromatic entangled light pulses. The scheme bases itself on the interaction between a bimodal cavity and the dipole of a trapped atom, which is driven by a laser pulse. EPR-type entanglement is established between the cavity modes via the quantum motion of the center of mass. As a result the light pulses emitted at the cavity output exhibit quantum correlations of EPR-type.

\section{A. Theoretical model}

We consider an atom of mass $m$, which is confined inside an optical cavity by an external potential, as shown in Fig. 1 . The center-of-mass motion is along the $\hat{x}$ axis, as we assume that the radial potential is sufficiently steep, that the motion in this plane can be considered frozen out. The potential along $\hat{x}$ is harmonic with frequency $\nu$. Position and momentum of the atomic center of mass are denoted by $x$ and $p$, respectively. The corresponding center-of-mass dynamics are given by

$$
H_{\mathrm{mec}}=\frac{p^{2}}{2 m}+\frac{1}{2} m \nu^{2} x^{2}=\hbar \nu\left(b^{\dagger} b+\frac{1}{2}\right),
$$

where $b, b^{\dagger}$ are the annihilation and creation operators, respectively, of a quantum of vibrational energy $\hbar \nu$, with $x$ $=\sqrt{\hbar / 2 m \nu}\left(b+b^{\dagger}\right)$ and $p=\mathrm{i} \sqrt{\hbar m \nu / 2}\left(b^{\dagger}-b\right)$. We denote by $\left|n_{\text {mec }}\right\rangle$ the eigenstates of $H_{\text {mec }}$ at energy $\hbar \nu\left(n_{\text {mec }}+1 / 2\right)$. The atom's relevant internal degrees of freedom are described by a ground state $|g\rangle$ and an excited state $|e\rangle$ which form a dipole with dipole moment $\mathbf{d}$ and frequency $\omega_{0}$, and the atomic Hamiltonian has the form

$$
H_{a}=\hbar \omega_{0}|e\rangle\langle e|+H_{\mathrm{mec}} .
$$

The full dynamics are described by Hamiltonian

$$
H=H_{a}+H_{c}+H_{a c}+H_{a L},
$$

where the terms $H_{c}, H_{a c}$, and $H_{a L}$ describe two cavity modes and the coupling of the atomic dipole to the electromagnetic field of the cavity and of a laser, respectively. The cavity Hamiltonian is

$$
H_{c}=\sum_{j=1,2} \hbar \omega_{j} a_{j}^{\dagger} a_{j},
$$

where $\omega_{j}$ are the frequencies of two optical modes, and $a_{j}, a_{j}^{\dagger}$ are the respective annihilation and creation operators of a quantum of energy $\hbar \omega_{j}$, i.e., a photon in mode $j$. We denote by $\left|n_{1}, n_{2}\right\rangle$ the eigenstates of $H_{c}$ at energy $\hbar \omega_{1} n_{1}+\hbar \omega_{2} n_{2}$. The coupling between the dipole and the cavity modes is represented by

$$
H_{a c}=\hbar \sum_{j=1,2} g_{j} a_{j} \sigma^{\dagger} \cos \left(k_{j} x \cos \theta_{c}+\phi_{j}\right)+\text { H.c. },
$$

whereby the modes have wave vectors $\vec{k}_{j}\left(k_{j}=\left|\vec{k}_{j}\right|\right)$ forming an angle $\theta_{c}$ with the axis $\hat{x}$ of the motion, and $g_{j}$ is the 
coupling strength of the dipole to the corresponding mode. The angle $\phi_{j}$ takes into account the position of the trap center inside the cavity. The terms $\sigma=|g\rangle\langle e|$ and $\sigma^{\dagger}=|e\rangle\langle g|$ denote the dipole lowering and raising operators. The coupling to the laser at frequency $\omega_{L}$ is

$$
H_{a L}=\hbar \Omega(t) \sigma^{\dagger} e^{-i\left(\omega_{L} t-k_{L} x \cos \theta_{L}\right)}+\text { H.c. , }
$$

where $\Omega$ is the (slowly varying) Rabi frequency and $\vec{k}_{L}$ is the wave vector $\left(k_{L}=\left|\vec{k}_{L}\right|\right)$ forming an angle $\theta_{L}$ with the trap axis. In what follows we drop the subscripts in the moduli of the wave vectors and denote them by $k$.

Denoting by $\rho$ the density matrix of the cavity modes and of the atom's internal and external degrees of freedom, the master equation for the dynamics reads

$$
\frac{\partial}{\partial t} \rho=\frac{1}{i \hbar}[H, \rho]+\mathcal{K} \rho+\mathcal{L} \rho
$$

which accounts for the coherent interaction of the dipole with the cavity modes, and the incoherent processes constituted by spontaneous emission and cavity decay, namely

$$
\begin{gathered}
\mathcal{L} \rho=\frac{\gamma}{2}\left(2 \sigma \widetilde{\rho} \sigma^{\dagger}-\sigma^{\dagger} \sigma \rho-\rho \sigma^{\dagger} \sigma\right), \\
\mathcal{K} \rho=\sum_{j=1,2} \kappa_{j}\left(2 a_{j} \rho a_{j}^{\dagger}-a_{j}^{\dagger} a_{j} \rho-\rho a_{j}^{\dagger} a_{j}\right),
\end{gathered}
$$

where $\gamma$ is the spontaneous emission rate of the atom into modes external to the cavity and $\kappa_{j}$ are the decay rates of the cavity modes. The density matrix $\tilde{\rho}$ accounts for the mechanical effect of photon emission,

$$
\tilde{\rho}=\int_{-1}^{1} d u \mathcal{N}(u) e^{-i k u x} \rho e^{i k u x}
$$

with probability $\mathcal{N}(u) d u$ that the spontaneously emitted photon imparts a recoil momentum $\hbar k u$ to the atom.

We assume that the atomic motion is in the Lamb-Dicke regime, and expand the interaction terms (4) and (5) to second order in the Lamb-Dicke parameter $\eta=k \sqrt{\hbar / 2 m \nu}$. In this limit they take the form

$$
\begin{aligned}
H_{a c}= & \hbar \sum_{j=1,2} g_{j} \cos \phi_{j}\left[a _ { j } \sigma ^ { \dagger } \left(1-\eta \cos \theta_{c} \tan \phi_{j}\left(b^{\dagger}+b\right)\right.\right. \\
& \left.\left.-\frac{\eta^{2}}{2} \cos ^{2} \theta_{c}\left(b^{\dagger}+b\right)^{2}\right)+\mathrm{O}\left(\eta^{3}\right)\right]+ \text { H.c. }
\end{aligned}
$$

and

$$
\begin{aligned}
H_{a L}= & \hbar \Omega(t) \sigma^{\dagger} e^{-i \omega_{L} t}\left(1+i \eta \cos \theta_{L}\left(b^{\dagger}+b\right)\right. \\
& \left.-\frac{\eta^{2}}{2} \cos ^{2} \theta_{L}\left(b^{\dagger}+b\right)^{2}+\mathrm{O}\left(\eta^{3}\right)\right)+ \text { H.c. }
\end{aligned}
$$

\section{B. Effective Hamiltonian}

We consider the reference frame rotating at the laser frequency, and denote by

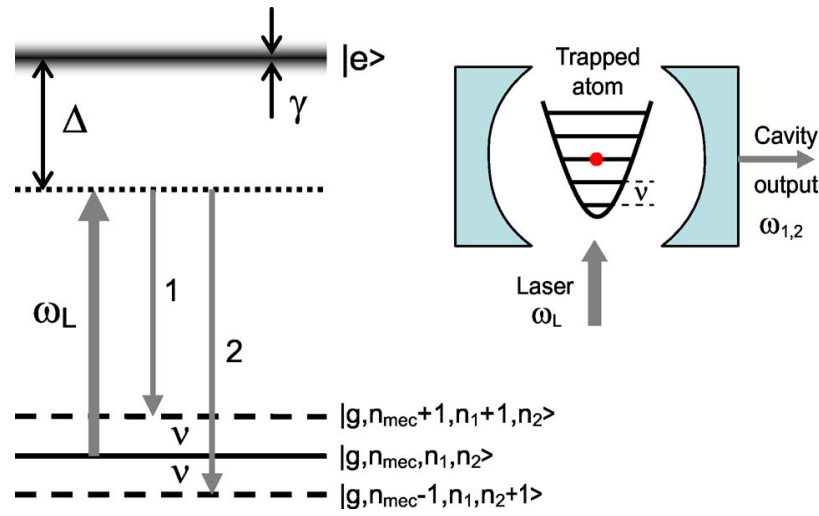

FIG. 2. (Color online) Layout of the system and energy diagram A single atom with internal energy levels $|g\rangle$ and $|e\rangle$ is confined by an external potential inside an optical cavity and is driven by a laser. The orientation of the considered vibrational mode has nonzero projection onto the laser direction. The harmonic motion, at frequency $\nu$, modulates the laser frequency, $\omega_{L}$, and the Stokes and anti-Stokes components at $\omega_{L} \pm \nu$ are resonant with two cavity modes, labeled 1 and 2. The linewidth of $|e\rangle$ is $\gamma$, and $\Delta$ is the detuning between laser and atom; $n_{\text {mec }}, n_{1}, n_{2}$ label the number of excitations of the center-of-mass, cavity mode 1 and cavity mode 2 oscillator, respectively.

$$
\Delta=\omega_{L}-\omega_{0}
$$

the detuning between laser and atom, and by

$$
\delta_{j}=\omega_{L}-\omega_{j}
$$

the detunings between the cavity modes and the laser. In particular, we assume

$$
\delta_{1}=\nu, \quad \delta_{2}=-\nu
$$

namely, the mode frequencies are spaced by the quantity $2 \nu$, and the laser frequency is tuned symmetrically between them. Hence, in this reference frame

$$
H_{c}^{\prime}=\hbar \nu\left(-a_{1}^{\dagger} a_{1}+a_{2}^{\dagger} a_{2}\right) .
$$

This choice of the frequency spacing and detunings allows us to select certain resonant scattering processes which determine the dynamics on the considered time scales. Figure 2 displays the quantum states which are resonantly coupled for this choice of the parameters. The corresponding effective Hamiltonian is derived in the following.

In the limit

$$
|\Delta| \gg \Omega, g_{j}, \gamma
$$

we eliminate the atom's internal degrees of freedom in second order perturbation theory in the parameter $\Omega /|\Delta|$ and obtain the approximate Hamiltonian,

$$
H_{\text {eff }}=H_{c}^{\prime}+H_{\text {mec }}+H_{1}+H_{2}
$$

which is defined on the subspace $\left|g, n_{\text {mec }}, n_{1}, n_{2}\right\rangle$. The dynamics of the coupling are given by the terms

$$
H_{1}=i \hbar \chi_{1} a_{1}^{\dagger} b^{\dagger}-i \hbar \tilde{\chi}_{1} a_{1} b,
$$




$$
H_{2}=i \hbar \chi_{2} a_{2}^{\dagger} b-i \hbar \widetilde{\chi}_{2} a_{2} b^{\dagger}
$$

with

$$
\begin{aligned}
& \chi_{1}=\eta g_{1}^{*} \cos \phi_{1} \Omega(t)\left(\frac{\cos \theta_{L}}{\Delta-\nu+i \gamma / 2}+\frac{i \tan \phi_{1} \cos \theta_{c}}{\Delta+i \gamma / 2}\right), \\
& \chi_{2}=\eta g_{2}^{*} \cos \phi_{2} \Omega(t)\left(\frac{\cos \theta_{L}}{\Delta+\nu+i \gamma / 2}+\frac{i \tan \phi_{2} \cos \theta_{c}}{\Delta+i \gamma / 2}\right),
\end{aligned}
$$

and

$$
\begin{aligned}
& \tilde{\chi}_{1}=\eta g_{1} \cos \phi_{1} \Omega(t)^{*}\left(\frac{\cos \theta_{L}}{\Delta-\nu+i \gamma / 2}-\frac{i \tan \phi_{1} \cos \theta_{c}}{\Delta+i \gamma / 2}\right), \\
& \tilde{\chi}_{2}=\eta g_{2} \cos \phi_{2} \Omega(t)^{*}\left(\frac{\cos \theta_{L}}{\Delta+\nu+i \gamma / 2}-\frac{i \tan \phi_{2} \cos \theta_{c}}{\Delta+i \gamma / 2}\right) .
\end{aligned}
$$

Hamiltonian (12) describes an interaction giving rise to twomode squeezing between the center-of-mass oscillator and the cavity mode at frequency $\omega_{1}$. Hamiltonian (13) describes a beam-splitter type of interaction between the center-ofmass oscillator and the cavity mode at frequency $\omega_{2}$ [27]. Their coupling strengths $\chi_{1}, \chi_{2}$, Eqs. (14) and (15), depend on the value of the atom-cavity coupling constants $g_{1}, g_{2}$, on the geometry of the setup, and on the ratio between the trap frequency $\nu$ and the laser detuning $\Delta$. In particular, each of them is the sum of two terms, which represent two indistinguishable paths leading to the creation of a cavity photon accompanied by the creation $\left(\chi_{1}\right)$ or annihilation $\left(\chi_{2}\right)$ of a vibrational quantum. This interference depends on the geometry of the setup and may lead to significantly different values of $\chi_{1}$ and $\chi_{2}$ when the ratio $\nu /|\Delta|$ is not too small $[32,33]$.

It should be remarked that these equations have been obtained at first order in the Lamb-Dicke expansion, neglecting off-resonant and inelastic scattering processes. They are valid on a time scale in which the resonant processes, in which a photon is scattered into the cavity mode under annihilation or creation of a vibrational quantum, dominate over all other processes. In addition, we have assumed that during these dynamics the cavity does not decay. This assumption implies that the duration of the laser pulses $T$ is much shorter than the cavity lifetime, $\kappa T \ll 1$. On the other hand, the dynamics are based on the spectral resolution of the cavity modes spaced by twice the trap frequency, i.e., $\nu T \gg 1$. Therefore, relation

$$
\kappa \ll \frac{1}{T} \ll \nu
$$

is required for the validity of the equations derived above. We refer the reader to Sec. II F for an extensive discussion of the parameter regimes in which Hamiltonian (11) holds. Note that the photons which are elastically scattered into modes external to the cavity do not affect the center-of-mass or cavity mode dynamics. Therefore, they can be traced out from the respective equations of motion without causing decoherence.

\section{Dynamics}

Let us now discuss the coherent physical dynamics that Eq. (11) describes, and neglect for the moment incoherent processes. In this case the observable $C=b^{\dagger} b-a_{1}^{\dagger} a_{1}+a_{2}^{\dagger} a_{2}$ is a constant of the motion. Therefore, if we consider the state $\left|n_{\text {mec }}, 0_{1}, 0_{2}\right\rangle$ at $t=0$, it will be coupled to states of the type $\left|n_{\text {mec }}+l_{1}-m_{2}, l_{1}, m_{2}\right\rangle$, which are eigenstates of $C$ at the same eigenvalue $n=n_{\text {mec }}$. The Heisenberg equations of motion

$$
\begin{gathered}
\dot{a}_{1}=\chi_{1} b^{\dagger}, \\
\dot{b}=\chi_{1} a_{1}^{\dagger}-\chi_{2}^{*} a_{2}, \\
\dot{a}_{2}=\chi_{2} b,
\end{gathered}
$$

generate periodic dynamics provided that $\left|\chi_{2}\right|>\left|\chi_{1}\right|$. In this case their solutions read [24]

$$
\begin{gathered}
a_{1}(t)=\frac{\chi_{1}}{\Theta} b^{\dagger}(0) \sin \Theta t+\frac{1}{\Theta^{2}}\left[\left|\chi_{2}\right|^{2}-\left|\chi_{1}\right|^{2} \cos \Theta t\right] a_{1}(0) \\
-\frac{\chi_{1} \chi_{2}}{\Theta^{2}}[1-\cos \Theta t] a_{2}^{\dagger}(0), \\
a_{2}(t)=\frac{\chi_{2}}{\Theta} b(0) \sin \Theta t+\frac{\chi_{1} \chi_{2}}{\Theta^{2}}[1-\cos \Theta t] a_{1}^{\dagger}(0) \\
\quad-\frac{1}{\Theta^{2}}\left[\left|\chi_{1}\right|^{2}-\left|\chi_{2}\right|^{2} \cos \Theta t\right] a_{2}(0), \\
b(t)=b(0) \cos \Theta t+\frac{1}{\Theta}\left[-\chi_{2}^{*} a_{2}(0)+\chi_{1} a_{1}^{\dagger}(0)\right] \sin \Theta t
\end{gathered}
$$

with

$$
\Theta=\sqrt{\left|\chi_{2}\right|^{2}-\left|\chi_{1}\right|^{2}} \text {. }
$$

In general these solutions describe tripartite entanglement among cavity modes and center-of-mass oscillator [24]. An interesting situation is found after half a period, for $T_{\pi}$ $=\pi / \Theta$. At this time (modulus $2 \pi$ ) we find

$$
\begin{gathered}
a_{1}\left(T_{\pi}\right)=\frac{\left|\chi_{1}\right|^{2}+\left|\chi_{2}\right|^{2}}{\Theta^{2}} a_{1}(0)-\frac{2 \chi_{1} \chi_{2}}{\Theta^{2}} a_{2}^{\dagger}(0), \\
a_{2}\left(T_{\pi}\right)=\frac{2 \chi_{1} \chi_{2}}{\Theta^{2}} a_{1}^{\dagger}(0)-\frac{\left|\chi_{1}\right|^{2}+\left|\chi_{2}\right|^{2}}{\Theta^{2}} a_{2}(0), \\
b\left(T_{\pi}\right)=-b(0) .
\end{gathered}
$$

Hence, at this instant the center-of-mass oscillator is decorrelated from the cavity modes. For instance, if at $t=0$ the center-of-mass oscillator density matrix is a thermal state at temperature $\mathcal{T}$ given by 


$$
\mu(0)=\left(1-e^{-\beta \hbar \nu}\right) e^{-\beta H_{\mathrm{mec}}},
$$

with $\beta=1 / k_{B} \mathcal{T}$, then $\mu\left(T_{\pi}\right)=\mu(0)$. Most remarkably, however, if at $t=0$ the cavity modes are in the vacuum, then at $t=T_{\pi}$ they exhibit EPR-type entanglement [2], their state being the two-mode squeezed state

$$
|\psi\rangle=\left(\frac{1-r^{2}}{1+r^{2}}\right) \sum_{n=0}^{\infty}\left(-\frac{2 r}{1+r^{2}} e^{i \phi}\right)^{n}|n, n\rangle,
$$

where

$$
r=\left|\frac{\chi_{2}}{\chi_{1}}\right|
$$

and $\phi=\arg \left(\chi_{1}\right)+\arg \left(\chi_{2}\right)$. The average number of photons per mode is

$$
\langle n\rangle=4 r^{2} /\left(1-r^{2}\right)^{2} .
$$

Hence, if the laser pulse has duration $T_{\pi}$, after the interaction the cavity modes are EPR-entangled with each other and decorrelated from the quantum state of the center-of-mass motion. The mechanical effects of the atom-photon interaction plays a fundamental role in establishing the entanglement, nevertheless the initial motional state does not affect the efficiency of the process.

\section{Field at the cavity output}

In this section we introduce the theoretical description of the field at the cavity output, which will be used in Sec. II E for determining the degree of quantum correlation of the emitted pulses. The cavity output is described by the Heisenberg operator [35]

$$
\mathbf{E}\left(x^{\prime}, t\right)=\mathbf{E}^{(+)}\left(x^{\prime}, t\right)+\mathbf{E}^{(-)}\left(x^{\prime}, t\right)
$$

where $\mathbf{E}^{(+)}\left(x^{\prime}, t\right)$ is the negative frequency part and $\mathbf{E}^{(-)}\left(x^{\prime}, t\right)$ its adjoint at the position $x^{\prime}$ outside the cavity, setting the mirror at $x^{\prime}=0$. We decompose the field into the free and the source field terms, according to

$$
\mathbf{E}^{(+)}\left(x^{\prime}, t\right)=\mathbf{E}_{\mathbf{s}}^{(+)}\left(x^{\prime}, t\right)+\mathbf{E}_{\mathbf{f}}^{(+)}\left(x^{\prime}, t\right),
$$

whereby the source field is given by

$$
\mathbf{E}_{\mathbf{s}}^{(+)}\left(x^{\prime}, t\right)=i \sum_{j} \hat{e}_{j} e^{i \phi_{T j}} \sqrt{\frac{\hbar \omega_{j}}{2 \epsilon_{0} A c}} \sqrt{2 \kappa_{j}} a_{j}\left(t-x^{\prime} / c\right),
$$

and only times $t>x^{\prime} / c$ are considered. Here, $A$ is the crosssectional area of the cavity mode, and $\phi_{T j}$ is the phase change on transmission through the output mirror. The free field is

$$
\mathbf{E}_{\mathbf{f}}^{(+)}\left(x^{\prime}, t\right)=i \sum_{j, k} \hat{e}_{j} \sqrt{\frac{\hbar \omega_{k}}{2 \epsilon_{0} A L^{\prime}}} r_{k}^{(j)}(0) e^{-i\left[\omega_{k}\left(t-x^{\prime} / c\right)-\phi_{R}\right]},
$$

which is defined for $x^{\prime}>0$. Here, $r_{k}^{(j)}$ and $r_{k}^{(j) \dagger}$ are annihilation and creation operators for the modes of the electromagnetic field external to the cavity at frequency $\omega_{k}$ and polarization $\hat{e}_{j} ; L^{\prime}$ is the quantization length at the cavity output, and $\phi_{R}$ the phase change upon reflection at the cavity output mirror.
Using

$$
r_{f}^{(j)}(t)=e^{i\left(\phi_{R}-\phi_{T j}\right)} \sum_{k} \sqrt{\frac{\omega_{k}}{\omega_{j}}} r_{k}^{(j)}(0) e^{-i \omega_{k} t}
$$

we introduce the rescaled field operator $Q_{j}^{(+)}\left(x^{\prime}, t\right)$ whereby

$$
Q_{j}^{(+)}\left(x^{\prime}, t\right)=\sqrt{c / L^{\prime}} r_{f}^{(j)}\left(t-x^{\prime} / c\right)+\sqrt{2 \kappa_{j}} a_{j}\left(t-x^{\prime} / c\right)
$$

such that [35]

$$
\mathbf{E}^{(+)}\left(x^{\prime}, t\right)=i \sum_{j} \hat{e}_{j} e^{i \phi_{T j}} \sqrt{\frac{\hbar \omega_{j}}{2 \epsilon_{0} A c}} Q_{j}^{(+)}\left(x^{\prime}, t\right),
$$

for $c t>x^{\prime}>0$. The decomposition of Eq. (35) shows how the photons transmitted through the mirror into the cavity output mix with the external fields reflected by the mirror itself.

Let us now discuss the dynamics of the cavity field, assuming that at $t=0$ a pulse of duration $T$ is applied which fulfills (16). At times $t>T$ the field inside the cavity evolves according to

$$
\begin{aligned}
a_{j}(T+t) \approx & a_{j}(T) e^{-\left(i \omega_{j}+\kappa_{j}\right) t} \\
& -\sqrt{\frac{c}{L^{\prime}}} \sqrt{2 \kappa_{j}} \int_{0}^{t} d \tau e^{-\left(i \omega_{j}+\kappa_{j}\right)(t-\tau)} r_{f}^{(j)}(\tau) .
\end{aligned}
$$

For later convenience, we generalize definition (35) and consider the rescaled field operator

$$
\begin{aligned}
Q_{j}\left(x^{\prime}, t, \theta_{j}\right) & =Q_{j}^{(+)}\left(x^{\prime}, t\right) e^{i \theta_{j}}+Q_{j}^{(-)}\left(x^{\prime}, t\right) e^{-i \theta_{j}} \\
& =\mathcal{Q}_{0}(t)\left[q_{j}\left(\theta_{j}\right)+\delta q_{j}\left(t, \theta_{j}\right)\right],
\end{aligned}
$$

where $\mathcal{Q}_{0}(t)=\sqrt{2 \kappa_{j}} e^{-\kappa_{j} t}$ is a time-dependent scalar, $q_{j}\left(\theta_{j}\right)$ is the cavity-field quadrature,

$$
q_{j}\left(\theta_{j}\right)=a_{j}(T) e^{i \theta_{j}}+a_{j}^{\dagger}(T) e^{-i \theta_{j}},
$$

and $\delta q_{j}\left(t, \theta_{j}\right)$ is the correspondingly defined quadrature of the free field.

\section{E. Correlations in the fields at the cavity output}

Quantum correlations in the two-mode output field $\mathbf{E}_{\text {out }}$ are detected by balanced homodyne detectors [2], using local oscillators $E_{1}^{(\mathrm{LO})}, E_{2}^{(\mathrm{LO})}$ with phases $\theta_{1}$ and $\theta_{2}$, respectively, which mix with the fields previously spatially separated by a beam splitter. A possible implementation is described in the following section. The measured currents at the detectors are $i_{1}(t)=\alpha Q_{1}\left(\theta_{1}\right)$ and $i_{2}(t)=\alpha Q_{2}\left(\theta_{2}\right)$, where $\alpha$ is a scaling parameter assumed to be equal for the two modes. The correlations are measured through the combined difference current $i_{-}(t)=i_{1}-i_{2}$, with

$$
i_{-}(t)=\alpha\left[Q_{1}\left(t, \theta_{1}\right)-Q_{2}\left(t, \theta_{2}\right)\right] .
$$

We evaluate the current fluctuations at time $t$ on a grid $\delta t$, such that $\kappa \delta t \ll 1$, i.e., fluctuations are recorded on a time scale much faster than the cavity decay time. The fluctuations of the difference current are given by

$$
\left\langle i_{-}(t)^{2}\right\rangle=i_{-}^{(0)} C_{1,2}(t),
$$

where 


$$
i_{-}^{(0)}=\alpha^{2}\left[\left\langle Q_{1}\left(t, \theta_{1}\right)^{2}\right\rangle+\left\langle Q_{2}\left(t, \theta_{2}\right)^{2}\right\rangle\right]
$$

is a positive proportionality constant, and

$$
C_{1,2}(t)=1-\frac{2\left\langle Q_{1}\left(t, \theta_{1}\right) Q_{2}\left(t, \theta_{2}\right)\right\rangle}{\left\langle Q_{1}\left(t, \theta_{1}\right)^{2}\right\rangle+\left\langle Q_{2}\left(t, \theta_{2}\right)^{2}\right\rangle}
$$

contains the effect of quantum correlations. In these equations the mean value $\langle\cdot\rangle$ of the operators at time $t$ is averaged over the interval of time $\delta t$ and the average is taken over the vacuum state of the electromagnetic field. Using Eqs. (32), (33), and (36), term (39) in the difference current (38) takes the form

$$
C_{1,2}(t)=1-\frac{\mathcal{R}(t)}{1+\mathcal{R}(t)} \frac{\left\langle q_{1}\left(\theta_{1}\right) q_{2}\left(\theta_{2}\right)\right\rangle}{\left\langle q_{1}\left(\theta_{1}\right)^{2}\right\rangle+\left\langle q_{2}\left(\theta_{2}\right)^{2}\right\rangle} .
$$

In Eq. (40) the relevant quantities are

$$
\mathcal{R}(t)=\kappa \delta t e^{-2 \kappa t}\left[\left\langle q_{1}\left(t, \theta_{1}\right)^{2}\right\rangle+\left\langle q_{2}\left(t, \theta_{2}\right)^{2}\right\rangle\right],
$$

and

$$
\begin{gathered}
\left\langle q_{1}\left(\theta_{1}\right)^{2}\right\rangle=\left\langle q_{2}\left(\theta_{2}\right)^{2}\right\rangle=\frac{\left(\left|\chi_{1}\right|^{2}+\left|\chi_{2}\right|^{2}\right)^{2}+4\left|\chi_{1} \chi_{2}\right|^{2}}{\Theta^{4}}, \\
\left\langle q_{1}\left(\theta_{1}\right) q_{2}\left(\theta_{2}\right)\right\rangle=\operatorname{Re}\left(\frac{4 \chi_{1} \chi_{2}\left(\left|\chi_{1}\right|^{2}+\left|\chi_{2}\right|^{2}\right)}{\Theta^{4}} e^{i\left(\theta_{1}+\theta_{2}\right)}\right) .
\end{gathered}
$$

The details of the derivation of this result are reported in the Appendix.

Let us now discuss function $C_{1,2}(t)$, Eq. (40), in detail. The second term on the right-handside of Eq. (40) is proportional to $\left\langle q_{1}\left(\theta_{1}\right) q_{2}\left(\theta_{2}\right)\right\rangle$ and gives the effect of quantum correlations. In absence of correlation between the two modes the average $\left\langle q_{1}\left(\theta_{1}\right) q_{2}\left(\theta_{2}\right)\right\rangle$ vanishes and $C_{1,2}(t)=1$. This value is the shot noise limit for independent vacuum inputs into the homodyne detectors.

The correlations $\delta\left(X_{1}-X_{2}\right)^{2}$ and $\delta\left(P_{1}+P_{2}\right)^{2}$ of the orthogonal quadratures $X$ and $P$ are obtained by setting $\theta_{1}$ $=\theta_{2}=0$ and $\theta_{1}=-\theta_{2}=\pi / 2$, respectively, which leads to identical results, namely

$$
C_{1,2}(t)=1-\frac{\mathcal{R}(t)}{1+\mathcal{R}(t)} \frac{2 r}{1+r^{2}} \frac{2}{1+\left[2 r /\left(1+r^{2}\right)\right]^{2}},
$$

where we have used definition (28). Thus, the regime $C_{1,2}(t)<1$ corresponds to detecting EPR-type entanglement $[2,4,34]$. Indeed, in this regime the value of $C_{1,2}(t)$ is an entanglement measure [34]. The effect on $C_{1,2}(t)$ of vacuum fluctuations that mix with the quantum correlations of the cavity field at the cavity output is represented by the parameter $\mathcal{R}(t)$, Eq. (41). This value is proportional to the number of photons inside the cavity, and goes to zero as a function of time on a scale determined by cavity decay. Therefore, for short times and large number of photons the effect of quantum correlations in the cavity field is well visible over the quantum noise. As the intensity of the source field diminishes with time, the signal reaches the shot noise limit.

Figure 3 shows the signal $C_{1,2}(t)$ for different values of the parameter $r$. A reduction below 10\% of the shot noise

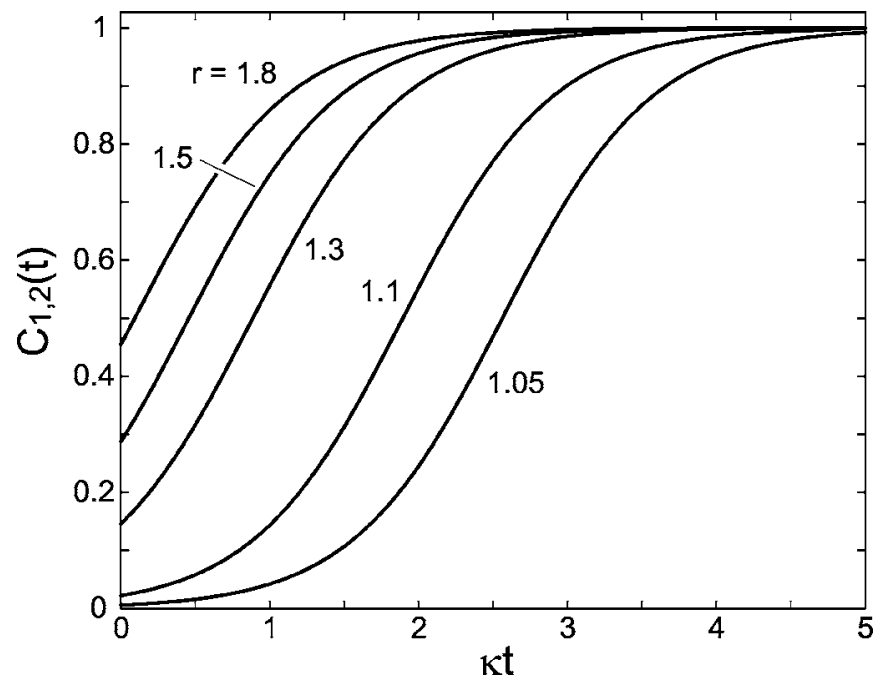

FIG. 3. Signal $C_{1,2}(t)$ as a function of time, for $\theta_{1}=\theta_{2}$ and for values of the parameter $r=1.8,1.5,1.3,1.1,1.05$ (from left to right). A time resolution of $\delta t=0.1 / \kappa$ is used. The other parameters are discussed in the text.

level is reached on a time scale of $1 / \kappa$ for $r=1.1$. About 110 photons per mode are created in this case. It should be noted that for $r$ close to 1, significant two-mode squeezing is observed over several cavity decay times, before the shot noise level $C_{1,2}(t)=1$ is approached. This occurs when the number of photons remaining in the cavity reaches the order of one.

\section{F. Experimental parameters}

We now discuss the parameter regime in which our description holds. First let us consider the ideal dynamics, as given by Eqs. (17)-(19). The degree of squeezing in the twomode state (27) is fixed by the ratio $r=\left|\chi_{2} / \chi_{1}\right|$, Eq. (28); large squeezing requires $r$ be close to 1 . With the cavity mode frequencies much larger than the trap frequency, we can assume $g_{1} \approx g_{2}=g$. Therefore, the degree of squeezing is solely controlled by the quantum effects in the mechanical action of the light, which enters through the ratio $\nu / \Delta$ between the trap frequency and the detuning (in this section we assume for convenience $\Delta$ for $|\Delta|)$. A small value of this ratio, i.e., $\nu \ll \Delta$, means large two-mode squeezing. Indeed, the control of the degree of squeezing through this ratio implies a further condition relating the linewidth of the transition $\gamma$ and the trap frequency $\nu$, namely

$$
\nu \gg \gamma,
$$

such that $\Delta \gg \nu \gg \gamma$. Under these conditions we find

$$
r \approx 1+2 \frac{\nu}{\Delta} .
$$

Furthermore we recall that $\Delta \gg \Omega, g$ was required throughout the model.

We now derive further conditions under which the dynamics are described by Eqs. (17)-(19). We have already identified in Eq. (16) an upper and a lower bound to the duration $T$ of the laser excitation pulse, due to cavity decay 
and to the spectral resolution of the cavity modes (whose frequency separation is fixed to twice the trap frequency). Other restrictions result from the requirement that processes in which the atom scatters laser photons into modes external to the cavity are negligible. Here some distinctions must be made. In fact, elastic scattering and inelastic scattering along the carrier (i.e., without changing the motion) do not affect the relevant dynamics, since they do neither change the number of phonons or cavity photons nor dephase their quantum states. Detrimental processes are (i) inelastic scattering of laser photons along the sidebands (i.e., changing the motion) and (ii) scattering of cavity photons into the external modes. Processes of type (i), which would add dissipation to Eq. (18), are characterized by a rate $\gamma_{\Theta} \sim \gamma \eta^{2} \Omega^{2} / \Delta^{2}$. During an excitation pulse of duration $T \sim 1 / \Theta$ they are negligible as long as

$$
\gamma_{\Theta} \ll \Theta .
$$

Processes of type (ii) occur at a rate $\gamma_{\kappa} \sim \gamma g^{2} / \Delta^{2}$. They enter as additional dissipative and noise terms into Eqs. (17) and (19), and are negligible provided that

$$
\gamma_{\kappa} \ll \kappa .
$$

Moreover, the coherent dynamics are based on the validity of the Lamb-Dicke regime at all times $0 \leqslant t \leqslant T$. This corresponds to the condition $\eta \sqrt{\left\langle b^{\dagger}(t) b(t)\right\rangle} \ll 1$ which can be rewritten as

$$
\eta \sqrt{\Delta / 4 \nu} \ll 1
$$

using Eqs. (22), (28), and (46). Finally, in the case of ion traps decoherence of the center-of-mass oscillation can safely be ignored, as the trapping potential has been experimentally demonstrated to be very stable on time scales of the order of milliseconds [36].

We now identify parameter regimes where significant shot noise reduction can be reached while conditions (16) and (47)-(49) are simultaneously fulfilled. We use [37]

$$
g=\sqrt{\frac{\sigma}{4 \pi A}} \sqrt{\gamma \delta \omega} \equiv \sqrt{\tilde{\sigma}} \sqrt{\gamma \delta \omega}
$$

where $\sigma \propto \lambda^{2}$ is the scattering cross section of the atom in free space, $A$ is the cavity mode waist, $L$ is the cavity length and $\delta \omega=2 \pi c / 2 L$ is the cavity free-spectral range. The cavity decay rate is $\kappa=\delta \omega / \mathcal{F}$ where $\mathcal{F}$ is the finesse. The condition $1 / T \gg \kappa$ in Eq. (16) together with $T=\pi / \Theta$ imposes the relation

$$
\frac{\Theta}{\kappa}=\sqrt{2} \eta \sqrt{\frac{2 \nu}{\Delta}} \frac{\Omega}{\Delta} \frac{g}{\kappa}=\mathcal{F} \sqrt{\frac{4 \nu \widetilde{\sigma}}{\Delta}}\left(\eta \frac{\Omega}{\Delta} \sqrt{\frac{\gamma}{\delta \omega}}\right) \gg 1,
$$

where we have used Eqs. (14) and (15) taking $\cos \theta_{L}=1$ and $\cos \theta_{c}=0$, i.e., the laser wave vector parallel to the motional axis, and the cavity wave vector perpendicular to both. Condition (47) leads to the relation

$$
\frac{\Theta}{\gamma_{\Theta}}=\sqrt{2} \sqrt{\frac{2 \nu}{\Delta}}\left(\eta \frac{\Omega}{\Delta}\right)^{-1} \frac{g}{\gamma}=\sqrt{\frac{4 \nu \tilde{\sigma}}{\Delta}}\left(\eta \frac{\Omega}{\Delta} \sqrt{\frac{\gamma}{\delta \omega}}\right)^{-1} \gg 1 .
$$

Finally, from (48) we find

$$
\frac{\kappa}{\gamma_{\kappa}}=\frac{\Delta^{2}}{\gamma^{2}} \frac{1}{\mathcal{F} \widetilde{\sigma}} \gg 1 .
$$

These inequalities can conveniently be summarized as

$$
\frac{4 \Delta \nu}{\gamma^{2}} \gg \mathcal{F} \frac{4 \nu \widetilde{\sigma}}{\Delta} \gg \sqrt{\frac{4 \nu \widetilde{\sigma}}{\Delta}}\left(\eta \frac{\Omega}{\Delta} \sqrt{\frac{\gamma}{\delta \omega}}\right)^{-1} \gg 1 .
$$

We consider now the ratio $\nu / \Delta=0.05$, which gives $r$ $=1.1$ [Eq. (46)], corresponding to significantly squeezed pulses with an average number of about 110 photons per mode. Taking realistic values $\eta=0.1, \Omega / \Delta=0.3$, and $\widetilde{\sigma}$ $=10^{-3}$, we obtain

$$
80\left(\frac{\nu}{\gamma}\right)^{2} \gg 2 \times 10^{-4} \mathcal{F} \gg 0.5 \sqrt{\frac{\delta \omega}{\gamma}} \gg 1 .
$$

Additionally, Eq. (45) is required; condition (49) is already met with the given choice of the Lamb-Dicke parameter $\eta$ and of the ratio $\nu / \Delta$.

A possible system to fulfil Eq. (54) and thus implement the desired dynamics is a single $\mathrm{In}^{+}$ion [38], confined by an ion trap of frequency $\nu=2 \pi \times 3 \mathrm{MHz}$, laser-excited on its intercombination line at $231 \mathrm{~nm}$ (linewidth $\gamma=2 \pi$ $\times 360 \mathrm{kHz})$ at $\Delta=2 \pi \times 60 \mathrm{MHz}$ detuning and $\Omega=2 \pi$ $\times 18 \mathrm{MHz}$ Rabi frequency, and coupled to an optical cavity with free spectral range $\delta \omega=2 \pi \times 1 \mathrm{GHz}$ and finesse $\mathcal{F}$ $=10^{6}$. For these parameters, $g \approx 2 \pi \times 0.6 \mathrm{MHz}, \Theta \approx 2 \pi$ $\times 8 \mathrm{kHz}$ and $\kappa \approx 2 \pi \times 1 \mathrm{kHz}$, and one would measure highly entangled pulses, characterized by $99 \%$ reduction of the vacuum fluctuations over a time of the order of $0.1 \mathrm{~ms}$.

To obtain a frequency splitting of $2 \nu \ll \delta \omega$ between the two cavity modes involved in the dynamics, two nondegenerate polarization modes may be utilized, both of which couple to the laser-driven transition of the atom. With $\mathrm{In}^{+}$, this is achieved by setting the quantization axis $\vec{B}$ along the cavity axis, and $\vec{B}, \vec{k}$, and laser polarization $\vec{E}_{L}$ mutually orthogonal. Other possible atomic level schemes are, e.g., a $J$ $=1 / 2 \leftrightarrow J^{\prime}=1 / 2$ or an $F=0 \leftrightarrow F^{\prime}=1$ transition. The twomode field emitted from the cavity after the laser excitation pulse is split by a polarizing beam splitter, and the fluctuations of both modes are detected by balanced homodyne detectors, as shown schematically in Fig. 4.

\section{TEMPORALLY SEPARATED ENTANGLED PULSES}

We now discuss a scheme which allows for the creation of pairs of temporally separated, entangled pulses, which may be monochromatic. The scheme is based on an atom trapped in a cavity, of which only one mode is relevant to the dynamics and that is far off-resonance from the dipole transition. The scheme is connected to ideas of quantum state transfer between the quantum center-of-mass motion and the 


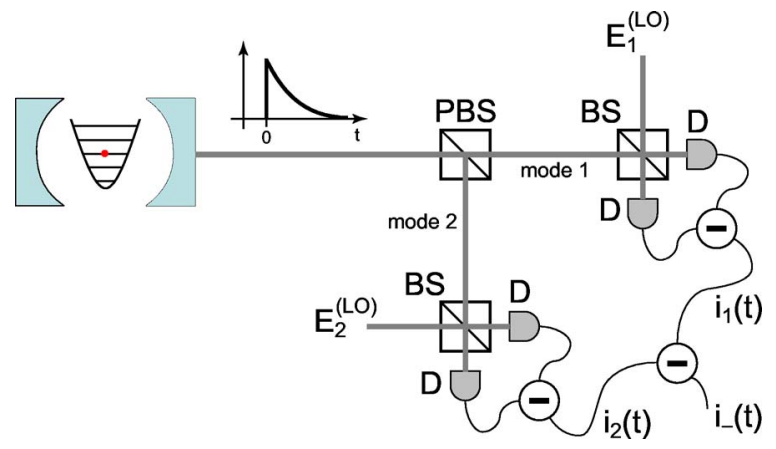

FIG. 4. (Color online) Schematic setup for measuring quantum correlations in the field at the cavity output. PBS stands for polarizing beam splitter, BS for beam splitter, $D$ for detector. Details of the experimental setup are discussed in the text.

electromagnetic field [27], and to its possible applications for creating quantum correlations between distant atoms [28]. In the present case, the ion keeps the memory of the quantum correlations with the field which is emitted by the cavity, and transfers it to the subsequent pulse. The resulting pulse pair exhibits EPR-type correlations in the quantum fluctuations.

\section{A. Dynamics}

We denote by $a, a^{\dagger}$ the annihilation and creation operator of a cavity photon at frequency $\omega_{c}$, and assume that both the center-of-mass and the cavity oscillator are initially prepared in the vacuum state. The model description is analogous to the one given in Sec. II A, whereby now the sum over the cavity modes is dropped, together with the subscript $j$.

The generation of pairs of monochromatic entangled pulses follows this procedure: first, a laser pulse is applied with $\omega_{L}=\omega_{c}+\nu$, in the regime in which the relevant dynamics are described by Hamiltonian $H_{\mathrm{eff}}^{(1)}=\hbar \omega_{c} a^{\dagger} a+H_{\mathrm{mec}}+H^{(1)}$ with

$$
H^{(1)}=i \hbar \chi a^{\dagger} b^{\dagger}+\text { H.c. },
$$

where the coupling term

$$
\chi=\eta \frac{g^{*} \cos \phi \Omega(t)}{\Delta}\left(\cos \theta_{L}+i \tan \phi \cos \theta_{c}\right)
$$

has been obtained in the limit $|\Delta| \gg \nu, \gamma$. Therefore, at the end of the laser pulse atomic motion and cavity mode are two-mode squeezed, their degree of squeezing being determined by the duration $T_{1}$ of the pulse according to

$$
\begin{gathered}
a\left(T_{1}\right)=a(0) \cosh |\chi| T_{1}+b^{\dagger}(0) e^{i \phi_{\chi} \sinh |\chi| T_{1},} \\
b^{\dagger}\left(T_{1}\right)=b^{\dagger}(0) \cosh |\chi| T_{1}+a(0) e^{-i \phi_{\chi} \sinh |\chi| T_{1},}
\end{gathered}
$$

with $\chi=e^{i \phi_{\chi}}|\chi|$. The average occupation number of both the cavity mode and the center-of-mass oscillator is $\langle n\rangle$ $=\sinh ^{2}|\chi| T_{1}$. The cavity field evolves according to Eq. (36) and after several decay times it is in the vacuum state, while the field at the cavity output is characterized by a propagating pulse described by Eq. (32), whose amplitude fluctuations are entangled with the motional state.
Let us then assume that at time $\tau \gg 1 / \kappa$ a second laser pulse is applied, which is now tuned to $\omega_{L}=\omega_{c}-\nu$, thus driving dynamics described by Hamiltonian $H_{\mathrm{eff}}^{(2)}=\hbar \omega_{c} a^{\dagger} a$ $+H_{\text {mec }}+H^{(2)}$ with

$$
H^{(2)}=i \hbar \chi a^{\dagger} b+\text { H.c. }
$$

at the same coupling constant $\chi$ as in Eq. (56). For a pulse duration $T_{2}=\pi / 2|\chi|$ then

$$
a\left(\tau+T_{2}\right)=b(\tau) e^{i \phi_{\chi}}
$$

and the motion is in the state of the cavity field at time $\tau$. In absence of decoherence processes for the atomic motion, $b(\tau) \propto b\left(T_{1}\right)$, whereby the proportionality factor is a timedependent phase which does not affect the efficiency of the scheme. Therefore, for $\kappa \tau \gg 1$, at the end of the second laser pulse the motion becomes decorrelated from the first propagating pulse and its correlations have been transferred to the cavity field, which is in a two-mode squeezed state with the first propagating pulse.

\section{B. Field at the cavity output}

The cavity field, entering Eq. (32) as the source field, is written as

$$
\begin{aligned}
a(t) \approx & \theta\left(t-T_{1}\right) a\left(T_{1}\right) e^{-\kappa t}+\theta\left(t-\tau-T_{2}\right) a\left(\tau+T_{2}\right) e^{-\kappa(t-\tau)} \\
& -\sqrt{\frac{c}{L^{\prime}}} \sqrt{2 \kappa} \int_{0}^{t} d \tau e^{-(i \omega+\kappa)(t-\tau)} r_{f}(\tau)
\end{aligned}
$$

and the source field (32) describes now two temporally separated pulses, whose separation can be controlled on a time scale of the order of the cavity decay time.

The correlation between the pulses can be detected by measuring the fluctuations of the difference current between the signals at the detector at $t$ and $t+\tau$, which we define as

$$
\tilde{i}_{-}(t, \tau)=\alpha\left[Q\left(t, \theta_{1}\right)-Q\left(t+\tau, \theta_{2}\right)\right] .
$$

The correlation function shows the same functional behaviour as function (44) where now $r$ is related to $|\chi|$ and $T_{1}$ by $\tanh |\chi| T_{1}=2 r /\left(1+r^{2}\right)$.

\section{Experimental parameters}

This type of proposal requires the coupling of the dipole with a single cavity mode, and it therefore simplifies several experimental conditions with respect to the simultaneous generation of bichromatic entangled pulses, see Sec. II. We list below some salient requirements.

Coherent dynamics during the laser pulse is achieved provided that $T_{1}, T_{2} \ll 1 / \kappa$. Moreover, spectral resolution of the vibrational excitations imposes $T_{1}, T_{2} \gg 1 / \nu$. Therefore, an important condition for the realization of this scheme is

$$
\nu \gg \frac{1}{T_{1}}, \frac{1}{T_{2}} \gg \kappa .
$$

This condition is accompanied by the requirements on negligible incoherent scattering by the atom, $\gamma_{\kappa} \ll \kappa$ [Eq. (48)] and $\gamma_{\Theta} T_{1} \ll 1$, which is equivalent to condition (47) for this 
type of scheme. Moreover, the Lamb-Dicke regime must be fulfilled at any stage of the dynamics. Therefore, large reductions in photon number correlations below the shot noise limit can be produced with atoms confined in very tight traps, i.e., with very small Lamb-Dicke parameters. This in turn affects the speed of the dynamics, as the coupling $\chi$ scales with $\eta$. For instance, after the first pulse the average number of vibrational excitations (and of cavity-mode photons) is $\langle n\rangle=\sinh ^{2}|\chi| T_{1}$, hence the Lamb-Dicke regime is fulfilled at all stages provided that

$$
\eta|\sinh | \chi\left|T_{1}\right| \ll 1 .
$$

If we set $\langle n\rangle \approx 100$, thus imposing $\eta=0.03$, then $T_{1}$ $\approx \ln 20 /|\chi|$. Taking these values, $\Omega / \Delta=0.3$, and $T_{2} \sim T_{1}$, we find from condition (60) a relation for the cavity parameters and the trap frequency,

$$
\nu \gg g / 300 \gg \kappa .
$$

Besides this, there is no particular requirement on the ratio $\gamma / \nu$, therefore these numbers can be obtained with various atomic species in experimentally available setups. It should be noticed that reliable entanglement between the temporally separated pulses requires that the coherence of the quantum state of the center-of-mass oscillator is preserved during and between the pulses. Generally, for ion traps one can rely on heating times of the order of tens of milliseconds, such that this condition is fulfilled [36]. A study of decoherence on the efficiency of the scheme will be the subject of future work.

Finally, we comment on the initial preparation of the center-of-mass state. The dynamics discussed here apply when the motion is prepared in the ground state of the confining potential, which may be achieved with ground state cooling techniques. However, initial preparation of the center-of-mass oscillator in the vacuum state is also possible by means of quantum state transfer techniques between the motion and the electromagnetic field [27]. Since these techniques are at the basis of the entanglement scheme, ground state cooling is not a necessary requirement. In future studies we will also investigate the scheme when the motion has been prepared in a different state than the ground state.

\section{CONCLUSIONS}

To conclude, we have shown that the motion of a single trapped atom inside an optical cavity can act as a quantum medium which mediates entanglement on demand between simultaneous or subsequent radiation pulses. The process is based on the mechanical effect of light, which in the quantum regime allows for coherently controlling the interaction and thereby the degree of entanglement. We have discussed two schemes, which allow for simultaneous bichromatic and temporally separated entangled pulses. From our estimates the proposal requires experimental regimes that are within reach, and would allow for the production of entangled light pulses on demand, characterized by $99 \%$ reduction of the vacuum fluctuations over a time of the order of $0.1 \mathrm{~ms}$.

Our schemes offer interesting alternatives to implementations with atomic gases $[3,7]$, where now the controlled in- teraction with the spectrum of the quantum excitations creates the entanglement with the radiation pulses. It can be extended to the microwave regime by suitably driving atomic microwave transitions in a setup like the one discussed in Ref. [39]. It can also be extended to the collective excitations of ultracold atomic gases, where the nature of the collective excitations would allow for additional freedom in tuning the parameters, thereby giving rise to higher efficiencies or new properties of the emitted radiation. The scheme with spatially separated entangled pulses may be of help in devising new cryptographic schemes exploiting time correlated pulses and continuous alphabets, thus extending those based on time-energy entangled photon pairs (see, e.g., Ref. [40] and references therein).

In the future we will study correlations in the continuouswave excitation of the ion, in the perspective of applications for quantum networking, like for instance discussed in Refs. $[41,42]$.

\section{ACKNOWLEDGMENTS}

The authors gratefully acknowledge discussions with Christoph Becher, Luiz Davidovich, Markus Hennrich, Paulo Nussenzveig, and Scott Parkins. This work was partly supported by the European Commission (CONQUEST network, MRTN-CT-2003-505089; SCALA, Contract No. 015714), and the scientific exchange programme Spain-Italy (HI20030075); one of the author (G. M.) is supported by the Spanish Ministerio de Educación y Ciencia (Ramon-y-Cajal and FIS2005-08257-C02-01).

\section{APPENDIX: EVALUATION OF THE FIELD CORRELATION FUNCTIONS}

In this appendix we report the detailed steps for the explicit derivation of the difference current (38). We assume $\kappa_{1} \approx \kappa_{2}$. The single terms on the right-hand side of Eq. (38) are evaluated to be

$$
\left\langle Q_{1}\left(t, \theta_{1}\right)^{2}\right\rangle=2 \kappa e^{-2 \kappa t}\left\langle q_{1}\left(\theta_{1}\right)^{2}\right\rangle+\frac{c}{L^{\prime}} \sum_{k} \frac{\omega_{k}^{(1)}}{\omega_{1}} \mathcal{I}_{1}(t),
$$

$$
\begin{gathered}
\left\langle Q_{2}\left(t, \theta_{2}\right)^{2}\right\rangle=2 \kappa e^{-2 \kappa t}\left\langle q_{2}\left(\theta_{2}\right)^{2}\right\rangle+\frac{c}{L^{\prime}} \sum_{k} \frac{\omega_{k}^{(2)}}{\omega_{2}} \mathcal{I}_{2}(t), \\
\left\langle Q_{1}\left(t, \theta_{1}\right) Q_{2}\left(t, \theta_{2}\right)\right\rangle=2 \kappa e^{-2 \kappa t}\left\langle q_{1}\left(\theta_{1}\right) q_{2}\left(\theta_{2}\right)\right\rangle,
\end{gathered}
$$

where

$$
\begin{aligned}
\left\langle q_{1}\left(\theta_{1}\right)^{2}\right\rangle=\left\langle\left(a_{1} e^{i \theta_{1}}+a_{1}^{\dagger} e^{-i \theta_{1}}\right)^{2}\right\rangle & =\frac{\left(\left|\chi_{1}\right|^{2}+\left|\chi_{2}\right|^{2}\right)^{2}+4\left|\chi_{1}\right|^{2}\left|\chi_{2}\right|^{2}}{\Theta^{4}}, \\
\left\langle q_{2}\left(\theta_{2}\right)^{2}\right\rangle & =\left\langle q_{1}\left(\theta_{1}\right)^{2}\right\rangle,
\end{aligned}
$$

and 


$$
\left\langle q_{1}\left(\theta_{1}\right) q_{2}\left(\theta_{2}\right)\right\rangle=\operatorname{Re}\left\{\frac{4 \chi_{1} \chi_{2}\left(\left|\chi_{1}\right|^{2}+\left|\chi_{2}\right|^{2}\right)}{\Theta^{4}} e^{i\left(\theta_{1}+\theta_{2}\right)}\right\},
$$

while the integral

$$
\mathcal{I}_{j}(t)=\frac{1}{\delta t^{2}} \int_{t}^{t+\delta t} d \tau \int_{t}^{t+\delta t} d \tau^{\prime} e^{-i \omega_{k}^{(j)}\left(\tau-\tau^{\prime}\right)}=2 \frac{\sin \omega_{k}^{(j)} \delta t}{\omega_{k}^{(j)} \delta t}
$$

introduces the finite spectral resolution associated with the temporal grid. With Eq. (A7) in Eqs. (A1) and (A2), we rewrite the sum over the free field modes as

$$
\sum_{k} \frac{\omega_{k}^{(j)}}{\omega_{j}} \mathcal{I}_{j}(t) \sim \frac{1}{\delta t},
$$

where we have taken the continuum limit of the discrete sum over the modes, thereby adding the density of states and assuming that $\omega_{k}^{(j)}$ varies negligibly over $1 / \delta t$. Substituting into Eqs. (A1) and (A2), we obtain

$$
\begin{aligned}
& \left\langle Q_{1}\left(t, \theta_{1}\right)^{2}\right\rangle=2 \kappa e^{-2 \kappa t}\left(\left\langle q_{1}\left(\theta_{1}\right)^{2}\right\rangle+\frac{e^{2 \kappa t}}{2 \kappa \delta t}\right), \\
& \left\langle Q_{2}\left(t, \theta_{2}\right)^{2}\right\rangle=2 \kappa e^{-2 \kappa t}\left(\left\langle q_{2}\left(\theta_{2}\right)^{2}\right\rangle+\frac{e^{2 \kappa t}}{2 \kappa \delta t}\right) .
\end{aligned}
$$

Taking $\omega_{1} \approx \omega_{2}$ we finally obtain

$$
C_{1,2}(t)=1-\frac{\mathcal{R}(t)}{1+\mathcal{R}(t)} c_{1,2}\left(\theta_{1}, \theta_{2}\right),
$$

where $\mathcal{R}(t)$ is defined in Eq. (41) and

$$
c_{1,2}\left(\theta_{1}, \theta_{2}\right)=\frac{2 \operatorname{Re}\left\{\left\langle q_{1}\left(\theta_{1}\right) q_{2}\left(\theta_{2}\right)\right\rangle\right\}}{\left\langle q_{1}\left(\theta_{1}\right)^{2}\right\rangle+\left\langle q_{2}\left(\theta_{2}\right)^{2}\right\rangle} .
$$

[1] S. L. Braunstein and P. van Loock, Rev. Mod. Phys. 77, 513 (2005).

[2] M. D. Reid, Phys. Rev. A 40, 913 (1989).

[3] A. Kuzmich, W. P. Bowen, A. D. Boozer, A. Boca, C. W. Chou, L.-M. Duan, and H. J. Kimble, Nature (London) 423, 731 (2003).

[4] V. Josse, A. Dantan, L. Vernac, A. Bramati, M. Pinard, and E. Giacobino, Phys. Rev. Lett. 91, 103601 (2003); V. Josse, A. Dantan, A. Bramati, M. Pinard, and E. Giacobino, ibid. 92, 123601 (2004).

[5] C. H. van der Wal, M. D. Eisaman, A. André, R. L. Walsworth, D. F. Phillips, A. S. Zibrov, and M. D. Lukin, Science 301, 196 (2003).

[6] M. D. Lukin, Rev. Mod. Phys. 75, 457 (2003).

[7] B. Julsgaard, J. Sherson, J. I. Cirac, J. Fiurasek, and E. S. Polzik, Nature (London) 432, 482 (2004).

[8] B. Blinov, D. L. Moehring, L.-M. Duan, and C. Monroe, Nature (London) 428, 153 (2004).

[9] J. Volz, M. Weber, D. Schlenk, W. Rosenfeld, J. Vrana, K. Saucke, C. Kurtsiefer, and H. Weinfurter, Phys. Rev. Lett. 96, 030404 (2006).

[10] C. W. Chou, H. de Riedmatten, D. Felinto, S. V. Polyakov, S. J. van Enk, and H. J. Kimble, Nature (London) 438, 828 (2005)

[11] K. An, J. J. Childs, R. R. Dasari, and M. S. Feld, Phys. Rev. Lett. 73, 3375 (1994).

[12] J. McKeever, A. Boca, A. D. Boozer, J. R. Buck, and H. J. Kimble, Nature (London) 425, 268 (2003).

[13] C. J. Hood, T. W. Lynn, A. C. Doherty, A. S. Parkins, and H. J. Kimble, Science 287, 1457 (2000).

[14] P. W. H. Pinkse, T. Fischer, P. Maunz, and G. Rempe, Nature (London) 404, 365 (2000).

[15] P. Bushev, A. Wilson, J. Eschner, C. Raab, F. Schmidt-Kaler, C. Becher, and R. Blatt, Phys. Rev. Lett. 92, 223602 (2004).

[16] A. B. Mundt, A. Kreuter, C. Becher, D. Leibfried, J. Eschner, F. Schmidt-Kaler, and R. Blatt, Phys. Rev. Lett. 89, 103001
(2002).

[17] G. R. Guthöhrlein, M. Keller, K. Hayasaka, W. Lange, and H. Walther, Nature (London) 414, 49 (2001).

[18] A. Kuhn, M. Hennrich, and G. Rempe, Phys. Rev. Lett. 89, 067901 (2002); T. Legero, T. Wilk, M. Hennrich, G. Rempe, and A. Kuhn, ibid. 93, 070503 (2004).

[19] J. McKeever, A. Boca, A. D. Boozer, R. Miller, J. R. Buck, A. Kuzmich, and H. J. Kimble, Science 303, 1992 (2004).

[20] M. Keller, B. Lange, K. Hayasaka, W. Lange, and H. Walther, Nature (London) 431, 1075 (2004).

[21] J. M. Raimond, M. Brune, and S. Haroche, Rev. Mod. Phys. 73, 565 (2001); B. T. H. Varcoe, S. Brattke, M. Weidinger, and H. Walther, Nature (London) 403, 743 (2000).

[22] G. Morigi, J. Eschner, S. Mancini, and D. Vitali, Phys. Rev. Lett. 96, 023601 (2006).

[23] S. Mancini, D. Vitali, and P. Tombesi, Phys. Rev. Lett. 90, 137901 (2003).

[24] S. Pirandola, S. Mancini, D. Vitali, and P. Tombesi, Phys. Rev. A 68, 062317 (2003).

[25] M. E. Anderson, D. F. McAlister, M. G. Raymer, and M. C. Gupta, J. Opt. Soc. Am. B 14, 3180 (1997); G. S. Kanter, P. Kumar, R. V. Roussev, J. Kurz, K. R. Parameswaran, and M. M. Fejer, Opt. Express 10, 177 (2002).

[26] C. Simon and D. Bouwmeester, Phys. Rev. Lett. 91, 053601 (2003).

[27] H. Zeng and F. Lin, Phys. Rev. A 50, R3589 (1994); A. S. Parkins and H. J. Kimble, J. Opt. B: Quantum Semiclassical Opt. 1, 496 (1999).

[28] A. Peng and A. S. Parkins, Phys. Rev. A 65, 062323 (2002).

[29] J. Ph. Karr, A. Baas, and E. Giacobino, Phys. Rev. A 69, 063804 (2004).

[30] M. Jakob and J. Bergou, Phys. Rev. A 60, 4179 (1999).

[31] R. Guzmán, J. C. Retamal, E. Solano, and N. Zagury, Phys. Rev. Lett. 96, 010502 (2006).

[32] J. I. Cirac, R. Blatt, A. S. Parkins, and P. Zoller, Phys. Rev. A 48, 2169 (1993); J. I. Cirac, M. Lewenstein, and P. Zoller, 
ibid. 51, 1650 (1995).

[33] S. Zippilli and G. Morigi, Phys. Rev. Lett. 95, 143001 (2005); Phys. Rev. A 72, 053408 (2005).

[34] G. Giedke, M. M. Wolf, O. Krüger, R. F. Werner, and J. I. Cirac, Phys. Rev. Lett. 91, 107901 (2003).

[35] H. J. Carmichael, An Open System Approach to Quantum Optics (Springer-Verlag, Berlin, Heidelberg, New York, 1991).

[36] For ion traps see for instance Q. A. Turchette, D. Kielpinski, B. E. King, D. Leibfried, D. M. Meekhof, C. J. Myatt, M. A. Rowe, C. A. Sackett, C. S. Wood, W. M. Itano, C. Monroe, and D. J. Wineland, Phys. Rev. A 61, 063418 (2000).

[37] H. J. Kimble, in Cavity Quantum Electrodynamics, edited by P.
R. Berman (Academic, New York, 1994), p. 203.

[38] E. Peik, J. Abel, Th. Becker, J. von Zanthier, and H. Walther, Phys. Rev. A 60, 439 (1999).

[39] F. Mintert and Ch. Wunderlich, Phys. Rev. Lett. 87, 257904 (2001); Ch. Wunderlich, G. Morigi, and D. Reiss, Phys. Rev. A 72, 023421 (2005).

[40] N. Gisin, G. Ribordy, W. Tittel, and H. Zbinden, Rev. Mod. Phys. 74, 145 (2002).

[41] J. I. Cirac, P. Zoller, H. J. Kimble, and H. Mabuchi, Phys. Rev. Lett. 78, 3221 (1997).

[42] B. Kraus and J. I. Cirac, Phys. Rev. Lett. 92, 013602 (2004). 